Pacific Journal of Mathematics

ÜBER EINE BEZIEHUNG ZWISCHEN MALCEV-ALGEBREN

OtTMAR Loos 


\title{
ÜBER EINE BEZIEHUNG ZWISCHEN MALCEV-ALGEBREN UND LIE-TRIPELSYSTEMEN
}

\section{OTTMAR LOOS}

\begin{abstract}
A Malcev algebra is an anticommutative algebra which satisfies the identity

$$
x y \cdot x z=(x y \cdot z) x+(y z \cdot x) x+(z x \cdot x) y .
$$
\end{abstract}

In this paper we construct to every Malcev algebra $A$ a Lie triple system $T_{A}$ and study the relations between them. A number of properties hold for a Malcev algebra if and only if they hold for the associated Lie triple system. E. g. the algebra $A$ is solvable (semisimple, simple) if and only if $T_{A}$ is. Moreover the radicals of $A$ and $T_{A}$ coincide. We shall prove:

Theorem A. A finite dimensional Malcev algebra A over a field of characteristics zero is semisimple if and only if the Killing form of $A$ is nondegenerate.

Let $C$ be the Cayley algebra over an algebraically closed field of characteristic zero. It has been shown by Sagle, that a simple 7-dimensional Malcev algebra $A^{*}$ can be obtained from $C$. Using a further theorem of Sagle we prove

Theorem B. Every simple finite dimensional non-Lie Malcev algebra over an algebraically closed field of characteristic zero is isomorphic to $A^{*}$.

2. A bezeichnet im folgenden stets, soweit nichts anderes gesagt wird, eine Malcev-Algebra endlicher Dimension über einem Körper der Charakteristik ungleich zwei. Es sei $L(x): y \rightarrow x y$ die Linksmultiplikation mit dem Element $x$ in $A$ und $(x, y)=$ Spur $L(x) L(y)$ die Killingform. Alle vorkommenden Vektorräume sind endlichdimensional. Für Elemente $x, y, z$ von $A$ sei

$$
J(x, y, z)=x y \cdot z+y z \cdot x+z x \cdot y
$$

Der Kommutator zweier linearer Transformationen $X$ und $Y$ wird mit $[X, Y]$ bezeichnet. Wir setzen

$$
D(x, y)=L(x y)+[L(x), L(y)]
$$

für $x, y$ aus $A$. Nach [4], Prop. 8.3 ist dies eine Derivation von $A$.

LEMMA 1. Fïr $x$, $y$ aus $A$ sei $R(x, y)=2 L(x y)+[L(x), L(y)]$. Dann gilt

$$
[L(z), R(x, y)]=R(z x, y)+R(x, z y)
$$


Beweis. Da $D(x, y)$ eine Derivation von $A$ ist, gilt

$$
L(D(x, y) z)=[D(x, y), L(z)] .
$$

Ferner gilt ([4], (2.12)):

$$
L(J(x, y, z))=[L(x), L(y z)]+[L(y), L(z x)]+[L(z), L(x y)] .
$$

Nun ist nach Definition $R(x, y)=L(x y)+D(x, y)$, daher

$$
\begin{aligned}
& {[L(z), R(x, y)]-R(z x, y)-R(x, z y)=[L(z), L(x y)]+[L(z), D(x, y)]} \\
& \quad-2 L(z x \cdot y)-[L(z x), L(y)]-2 L(x \cdot z y)-[L(x), L(z y)] \\
& \quad=L(-D(x, y) z-2 z x \cdot y-2 x \cdot z y)+L(J(x, y, z)) \\
& \quad=L(-x y \cdot z-x \cdot y z+y \cdot x z-2 z x \cdot y-2 x \cdot z y)+L(J(x, y, z))=0 .
\end{aligned}
$$

3. Wir erinnern daran, dass ein Lie-Tripelsystem ein Vektorraum $T$ mit einer trilinearen Verknüpfung $[x, y, z]$ ist, die den folgenden Bedingungen genügt:

$$
\begin{array}{cc}
\text { (1) } & {[x, x, y]=0 .} \\
\text { (2) } & {[x, y, z]+[y, z, x]+[z, x, y]=0 .} \\
\text { (3) } & {[u, v,[x, y, z]]=[[u, v, x], y, z]+[x,[u, v, y], z]+[x, y,[u, v, z]] .}
\end{array}
$$

Eine lineare Abbildung $D$ von $T$ in sich heisst eine Derivation des Lie-Tripelsystems, wenn

$$
D[x, y, z]=[D x, y, z]+[x, D y, z]+[x, y, D z] .
$$

Die Bedingung (3) ist damit äquivalent, dass die Abbildung $z \rightarrow[x, y, z]$ eine Derivation von $T$ ist. Wir bezeichnen diese Abbildung ebenfalls mit $R(x, y)$. Die Rechtfertigung dafür gibt

SAtz 1. Sei A eine Malcev-Algebra. Mit der Komposition

$$
[x, y, z]=R(x, y) z=2 x y \cdot z-y z \cdot x-z x \cdot y
$$

ist $A$ ein Lie-Tripelsystem. Jedes $L(x)$ ist eine Derivation dieses Lie-Tripelsystems.

Beweis. Die Bedingung (1) ist klar. Zum Beweis von (2) bemerken wir, dass $J(x, y, z)$ invariant gegen zyklische Vertauschung ist, daher

$$
[x, y, z]+[y, z, x]+[z, x, y]=2 J(x, y, z)-J(y, z, x)-J(z, x, y)=0 .
$$

Lemma 1 besagt gerade, dass jedes $L(x)$ eine Derivation der Verknüpfung $[x, y, z]$ ist. Dasselbe gilt für $D(x, y)$; denn dies ist sogar eine Derivation von $A$. Daher ist auch $R(x, y)=L(x y)+D(x, y)$ 
eine Derivation der dreistelligen Verknüpfung $[x, y, z]$, also ist $A$ ein Lie-Tripelsystem.

Das derart jeder Malcev-Algebra zugeordnete Lie-Tripelsystem wird mit $T_{A}$ bezeichnet. Man beachte, dass $A$ und $T_{A}$ denselben zu Grunde liegenden Vektorraum haben. Offenbar ist jede Derivation von $A$ auch eine Derivation von $T_{A}$.

4. In einem beliebigen Lie-Tripelsystem $T$ sei $\mathscr{D}_{0}(T)$ die Menge der Derivationen der Form $\sum R\left(x_{i}, y_{i}\right)$ (innere Derivationen) und $\mathscr{D}(T)$ die Lie-Algebra aller Derivationen von $T$. Die direkte Vektorraumsumme $T \oplus \mathscr{D}(T)$ wird mit den Verknüpfungen

$$
\begin{aligned}
{[x, y] } & =R(x, y) \\
{[D, x] } & =-[x, D]=D x \\
{\left[D_{1}, D_{2}\right] } & =D_{1} D_{2}-D_{2} D_{1}
\end{aligned}
$$

für $x, y$ aus $T$ und $D, D_{1}, D_{2}$ aus $\mathscr{D}(T)$ zu einer Lie-Algebra. $T \oplus \mathscr{D}_{0}(T)$ ist ein Ideal in $T \oplus \mathscr{D}(T)$ und es gilt $[T, T]=\mathscr{D}_{0}(T)$. Die Lie-Algebra $T \oplus \mathscr{D}_{0}(T)=T \oplus[T, T]$ heisst die Standard-Einbettung von $T([8])$. Ein Unterraum $V$ von $T$ heisst ein Ideal des LieTripelsystems, wenn $[V, T, T] \subset V$. Dabei bezeichnet $[V, T, T]$ den von allen Elementen der Form $[v, x, y]$ mit $v$ aus $V$ und $x, y$ aus $T$ erzeugten linearen Unterraum von $T$. Aus der Jakobi-Identität (2) folgt

$$
[T, T, V] \subset[T, V, T]+[V, T, T]=[V, T, T] \subset V ;
$$

$V$ ist also unter allen inneren Derivationen invariant. Ist $V$ sogar unter allen Derivationen invariant, so heisst es ein charakteristisches Ideal.

Lemma 2. Sei A eine Malcev-Algebra und $T_{A}$ das zugehörige Lie-Tripelsystem. $\mathscr{L}(A)$ sei die von allen $L(x), x \in A$, erzeugte LieAlgebra. Dann ist

$$
\mathscr{D}_{0}\left(T_{A}\right) \subset \mathscr{L}(A) \subset \mathscr{D}\left(T_{A}\right) .
$$

Jedes Ideal von $A$ ist, als Unterraum von $T_{A}$ betrachtet, ein Ideal von $T_{A}$. Jedes charakteristische Ideal von $T_{A}$ ist als Unterraum von $A$ ein Ideal von $A$.

Beweis. Jedes Element von $\mathscr{D}_{0}\left(T_{A}\right)$ ist Summe von Transformationen der Form $2 L(x y)+[L(x), L(y)]$; daher die erste Inklusion. Nach Satz 1 ist jedes $L(x)$ eine Derivation von $T_{A}$; daher die zweite 
Inklusion. Wie aus der Definition der Verknüpfung in $T_{A}$ unmittelbar folgt, ist ein Ideal in $A$ als Unterraum von $T_{A}$ ein Ideal von $T_{A}$. Die Ideale von $A$ sind genau die unter $\mathscr{L}(A)$ invarianten Unterräume, daher folgt aus $\mathscr{L}(A) \subset \mathscr{D}\left(T_{A}\right)$ die letzte Behauptung.

Bemerkung. Wie aus dem Beweis hervorgeht, ist bereits ein charakteristischer Unterraum von $T_{A}$, d.h. ein Unterraum, der unter allen Derivationen von $T_{A}$ invariant ist, ein Ideal von $A$ und damit auch von $T_{A}$.

5. In einer beliebigen nicht assoziativen Algebra $A$ sei

$$
A^{(0)}=A ; A^{(k+1)}=A^{(k)} \cdot A^{(k)} .
$$

A heisst auflösbar, wenn es ein $k$ gibt, sodass $A^{(k)}=0$. Es ist wohlbekannt ([7]), dass Unteralgebren und homomorphe Bilder auflösbarer Algebren wieder auflösbar sind. Ist ferner $B$ ein auflösbares Ideal in $A$ und $A / B$ auflösbar, so ist auch $A$ auflösbar ([7], Prop. 1, p. 17). Sind $B$ und $C$ auflösbare Ideale in $A$, so ist auch $B+C$ ein aufösbares Ideal; denn $B$ und $(B+C) / B \cong B /(B \cap C)$ sind auflösbar. In jeder nicht assoziativen Algebra gibt es daher ein maximales aufiösbares Ideal.

Definition. Das Radikal einer Malcev-Algebra ist ihr maximales auflösbares Ideal. Die Algebra heisst halbeinfach, wenn ihr Radikal Null ist.

Wir nennen eine Algebra einfach, wenn sie keine nichttrivialen Ideale hat und $A^{2} \neq 0$ ist. Es ist klar, dass eine einfache MalcevAlgebra halbeinfach ist.

Lemma 3. Das Radikal $R$ einer Malcev-Algebra A ist das kleinste Ideal, sodass $A / R$ halbeinfach ist.

Beweis. Siehe [7], Prop. 2, p. 17.

Lemma 4. Sind $A_{1}, \cdots, A_{n}$ Malcev-Algebren, so ist das Radikal der direkten Summe der $A_{i}$ die direkte Summe der Radikale $R_{i}$ der $A_{i}$.

Beweis. Die Summe $R^{\prime}$ der $R_{i}$ ist ein auflösbares Ideal in $A$, also $R^{\prime} \subset R$. Die Projektionen von $R$ in die $A_{i}$ sind auflösbare Ideale in $A_{i}$ und daher in $R_{i}$ enthalten; also $R \subset R^{\prime}$.

6. In diesem Abschnitt setzen wir voraus, dass der Grundkörper die Charakteristik Null hat. Wir wiederholen einige bekannte Tatsachen 
über Lie-Tripelsysteme, vgl. dazu [3]. Sei $V$ ein Ideal eines LieTripelsystems $T$. Wir setzen

$$
D^{\circ} V=V ; D^{K+1} V=\left[T, D^{K} V, D^{K} V\right] \text {. }
$$

$V$ heisst auflösbar, wenn es ein $k$ gibt, sodass $D^{K} V=0$. Das maximale auflösbare Ideal von $T$ existiert, es heisst das Radikal von T. $T$ heisst halbeinfach, wenn das Radikal Null ist. Hat $T$ nur die trivialen Ideale 0 und $T$, so heisst es einfach.

Lemma 5. Das Radikal eines Lie-Tripelsystems $T$ über einem Körper der Charakteristik Null ist ein charakteristisches Ideal.

Beweis. Das Radikal der Lie-Algebra $T \oplus \mathscr{D}_{0}(T)=T \oplus[T, T]$ ist $R \oplus[R, T]$ ([3], Th. 2.16). $T \oplus[T, T]$ ist ein Ideal in $T \oplus \mathscr{D}(T)$, daher ist für jedes $D$ aus $\mathscr{D}(T)$ die Linksmultiplikation mit $D$ in $T \oplus \mathscr{D}(T)$ eine Derivation von $T \oplus[T, T]$. Das Radikal einer LieAlgebra ist ein charakteristisches Ideal ([1]), daher folgt aus der Definition der Multiplikation in $T \oplus \mathscr{D}(T)$ (vgl. 4.), dass $D R \subset R$.

SATz 2. Sei $A$ eine Malcev-Algebra über einem Körper der Charakteristik Null und $T_{A}$ das zugehörige Lie-Tripelsystem. Die Radikale von $A$ und $T_{A}$ stimmen überein. Insbesondere ist $A$ genau dann halbeinfach, wenn $T_{A}$ halbeinfach ist.

Beweis. Sei $B$ ein Ideal von $A$, das als Ideal des Lie-Tripelsystems $T_{A}$ auflösbar ist. Sei etwa $D^{k} B=0$. Wir zeigen durch Induktion nach $k$, dass $B$ ein auflösbares Ideal von $A$ ist.

Induktionsanfang. $\quad D^{1} B=\left[T_{A}, B, B\right]=0$. Dann ist für $x, y, z$ aus

$$
B: \quad[x, y, z]=R(x, y) z=(2 L(x y)+[L(x), L(y)]) z=0
$$

oder $L(x y) z=-D(x, y) z$. Da $D(x, y)$ eine Derivation von $A$ ist, ist die Einschränkung von $L(x y)$ auf $B$ eine Derivation von $B$; es gilt also für $u, v$ aus $B$ :

$$
x y \cdot u v=(x y \cdot u) v+u(x y \cdot v),
$$

und demnach

$$
\begin{aligned}
0 & =[u, v, x y]=2 u v \cdot x y-(v \cdot x y) u-(x y \cdot u) v \\
& =3 u v \cdot x y+x y \cdot u v-(x y \cdot u) v-u(x y \cdot v)=3 u v \cdot x y,
\end{aligned}
$$

das heisst $B^{2} \cdot B^{2}=B^{(2)}=0$ und $B$ ist auflösbar.

Sei die Behauptung schon bewiesen für Ideale $B$ von $A$ mit 
$D^{k} B=0$ und sei $B$ ein Ideal von $A$ mit $D^{k+1} B=0$. Da jedes $L(x)$ eine Derivation von $T_{A}$ ist (Satz 1 ), gilt

$$
L(x)\left(D^{1} B\right)=\left[x T_{A}, B, B\right]+\left[T_{A}, x B, B\right]+\left[T_{A}, B, x B\right] \subset\left[T_{A}, B, B\right],
$$

also ist $D^{1} B$ ein Ideal von $A$. Wegen $[B, B, B] \subset D^{1} B$ ist $B / D^{1} B$ eine Malcev-Algebra, deren zugehöriges Lie-Tripelsystem trivial ist. Nach Induktionsanfang ist daher $B / D^{1} B$ eine auflösbare Malcev-Algebra. Ferner ist $D^{k}\left(D^{1} B\right)=D^{k+1} B=0$, also ist nach Induktionsannahme $D^{1} B$ auflösbar. Daher ist auch $B$ auflösbar (vgl. 5.).

Da das Radikal $R_{T}$ von $T_{A}$ ein charakterisches Ideal von $T_{A}$ (Lemma 5 ) und deshalb ein Ideal von $A$ ist (Lemma 2), folgt, dass es im Radikal $R$ von $A$ enthalten ist. Um die umgekehrte Inklusion $\mathrm{zu}$ beweisen, zeigen wir zunächst: Ist $T_{A}$ ein halbeinfaches Lie-Tripelsystem, so ist $A$ eine halbeinfache Malcev-Algebra. In einem halbeinfachen LieTripelsystem ist jede Derivation eine innere Derivation und die LieAlgebra $\mathscr{D}\left(T_{A}\right)$ der Derivationen von $T_{A}$ operiert vollständig reduzibel auf $T_{A}$ ([3], Th. 2.11 und Th. 4.1). Nach Lemma 2 folgt $\mathscr{D}_{0}\left(T_{A}\right)=$ $\mathscr{L}(A)=\mathscr{D}\left(T_{A}\right)$ und daher operiert $\mathscr{L}(A)$ vollständig reduzibel auf $A$. Da die Ideale von $A$ genau die unter $\mathscr{L}(A)$ invarianten Unterräume sind, ist $A$ direkte Summe von Idealen, die einfache $\mathscr{L}(A)$ Moduln sind. Sei $I$ eines dieser Ideale. Ist $I^{2}=0$, so gibt es ein Ideal $I^{\prime}$ mit $I \oplus I^{\prime}=A$ und wegen $I \cdot I^{\prime}=0$ folgt $I \cdot A=0$. Es folgt $D^{1} I=\left[T_{A}, I, I\right]=0$, also ist $I$ ein auflösbares Ideal von $T_{A}$ und daher $I \subset R_{T}=0 . A$ ist also direkte Summe einfacher Ideale. Da das Radikal einer einfachen Algebra Null ist und das Radikal einer direkten Summe gleich der direkten Summe der Radikale der Summanden ist (Lemma 4), ist das Radikal von $A$ Null und daher $A$ halbeinfach.

$A / R_{T^{\prime}}=T_{\mathbb{A}} / R_{T}$ ist ein halbeinfaches Lie-Tripelsystem und daher nach dem eben Bewiesenen eine halbeinfache Malcev-Algebra. Aus Lemma 3 folgt dann $R \subset R_{r}$ und zusammen mit der schon bewiesenen Inklusion: $R=R_{r}$.

7. Alle folgenden Korollare gelten für einen Grundkörper der Charakteristik Null. Aus dem Beweis von Satz 2 folgt unmittelbar:

Korollar 1. Eine Malcev-Algebra ist genau dann halbeinfach, wenn sie direkte Summe einfacher Ideale ist.

Bemerkung. Sagle [4] hat das Radikal einer Malcev-Algebra als das kleinste Ideal definiert, sodass die Quotientenalgebra Summe einfacher Ideale ist. Wie Korollar 1 und Lemma 3 zeigen, stimmt diese Definition mit der unsrigen überein.

Korollar 2. In einer halbeinfachen Malcev-Algebra ist ein 
Unterraum genau dann ein Ideal, wenn er ein Ideal im zugehörigen Lie-Tripelsystem ist. Insbesondere ist A genau dann einfach, wenn $T_{A}$ einfach ist.

Beweis. Wegen Lemma 2 bleibt zu zeigen, dass jedes Ideal von $T_{A}$ auch ein Ideal von $A$ ist. Da $T_{A}$ halbeinfach ist, ist ein Ideal von $T_{A}$ unter $\mathscr{D}_{0}\left(T_{A}\right)=\mathscr{L}(A)=\mathscr{D}\left(T_{A}\right)$ invariant, also ein Ideal von $A$.

Eine Derivation von $A$ heisst eine innere Derivation, wenn sie in $\mathscr{L}(A)$ enthalten ist ([4]). Das folgende Korollar ist von Sagle [6] auf anderem Wege bewiesen worden.

KOROLLAR 3. In einer halbeinfachen Malcev-Algebra ist jede Derivation eine innere Derivation.

Beweis. Da jede Derivation von $A$ auch eine Derivation von $T_{A}$ ist, folgt dies aus $\mathscr{L}(A)=\mathscr{D}\left(T_{A}\right)$.

Korollar 4. Das Radikal einer Malcev-Algebra ist ein charakteristisches Ideal; das heisst, es ist unter allen Derivationen von $A$ invariant.

Beweis. $\quad R=R_{r}$ ist sogar unter allen Derivationen von $T_{A}$ invariant (Lemma 5 ).

Sei $V$ ein Ideal von $T_{\Delta}$. Wir setzen $V_{(0)}=V ; V_{(k+1)}=\left[V_{(k)}, V_{(k)}, V_{(k)}\right]$ und nennen $V$ schwach auflösbar, wenn $V_{(k)}=0$ für genügend grosses $k$. Wegen $V_{(k)} \subset D^{k} V$ ist jedes auflösbare Ideal von $T_{A}$ auch schwach auflösbar.

Korollar 5. Sei $B$ ein Ideal von $A$. Ist $B$ auflösbar, so ist es als Ideal von $T_{A}$ auflösbar. Ist $B$ als Ideal von $T_{A}$ schwach auflösbar, so ist es als Ideal von $A$ auflösbar.

Beweis. Ist $B$ auflösbar, so ist $B \subset R=R_{x^{\prime}}$ und wegen $D^{k} B \subset$ $D^{k} R_{T}$ ist $B$ als Ideal von $T_{A}$ auflösbar. Wie der Beweis von Satz 2 ergibt, ist $B$ als schwach auflösbares Ideal von $T_{A}$ als Ideal von $A$ auflösbar.

8. Ist $\mathscr{L}$ eine Lie-Algebra mit einem involutorischen Automorphismus $\sigma$ über einem Körper der Charakteristik $\neq 2$ und $\mathscr{L}=$ $\mathscr{L}_{+} \oplus \mathscr{L}_{-}$die Zerlegung von $\mathscr{L}$ in die Eigenräume zum Eigenwert \pm 1 von $\sigma$, so bildet $\mathscr{L}_{-}$ein Lie-Tripelsystem bezüglich der Verknüpfung $[x, y, z]=[[x, y], z]$. Es gelten die Regeln 


$$
\left[\mathscr{L}_{+}, \mathscr{L}_{+}\right] \subset \mathscr{L}_{+} ;\left[\mathscr{L}_{+}, \mathscr{L}_{-}\right] \subset \mathscr{L}_{-} ;\left[\mathscr{L}_{-}, \mathscr{L}_{-}\right] \subset \mathscr{L}_{+} .
$$

Lemma 6. Sei A eine Malcev-Algebra und $T_{A}$ das zugehörige Lie-Tripelsystem. $T_{A}$ sei als Unterraum der Elemente zum Eigenwert -1 bezüglich eines involutorischen Automorphismus $\sigma$ in eine Lie-Algebra $\mathscr{L}$ eingebettet. Sei $\beta$ die Killingform von $\mathscr{L}$ und $(x, y)=\operatorname{Sp} L(x) L(y)$ die Killingform von $A$. Dann gilt für $x, y$ aus $A$ :

$$
6(x, y)=\beta(x, y)
$$

Beweis. Seien $x_{1}, \cdots, x_{k}$ bzw. $y_{1}, \cdots, y_{l}$ Basen von $T_{A}=\mathscr{L}_{-}$bzw. $\mathscr{L}_{+}$. Mit ad $x$ bezeichnen wir die Linksmultiplikation mit $x$ in $\mathscr{L}$. Für $x$ aus $T_{A}$ ist dann

$$
\text { ad } x \cdot x_{i}=\sum \alpha_{i \mu} y_{\mu} ; \quad \text { ad } x \cdot y_{\mu}=\sum \beta_{\mu k} x_{k} ;
$$

und

$$
(\operatorname{ad} x)^{2} \cdot x_{i}=\sum \alpha_{i \mu} \beta_{\mu l k} x_{k} ; \quad(\operatorname{ad} x)^{2} \cdot y_{\mu}=\sum \beta_{\mu k} \alpha_{k \lambda} y_{\lambda} ;
$$

daher ist $\beta(x, x)=2 \sum \alpha_{i \mu} \beta_{\mu i}$. Andrerseits ist

$$
\begin{aligned}
(\operatorname{ad} x)^{2} \cdot x_{i} & =\left[x,\left[x, x_{i}\right]\right]=\left[\left[x_{i}, x\right], x\right]=\left[x_{i}, x, x\right] \\
& =2 x_{i} x \cdot x-x x_{i} \cdot x=3 x \cdot x x_{i}=3 L(x)^{2} \cdot x_{i} ;
\end{aligned}
$$

also

$$
3(x, x)=3 \operatorname{Sp} L(x)^{2}=\sum \alpha_{i \mu} \beta_{\mu i}=1 / 2\{\beta(x, x)\} .
$$

Durch Polarisation folgt die Behauptung.

Ist $V$ ein linearer Unterraum von $A$, so bezeichnen wir mit $V^{\perp}$ den zu $V$ bezüglich der Killingform orthogonalen Unteraum, also $V^{\perp}=\{x \in A: \quad(x, y)=0$ für alle $y$ aus $V\}$.

SATZ 3. Sei A eine Malcev-Algebra über einem Körper der Charakteristik Null und $R$ ihr Radikal. Dann gilt

$$
R=\left(A^{(k)}\right)^{\perp} \quad \text { für } k \geqq 2 .
$$

Beweis. Wir setzen $\mathscr{L}=T_{A} \oplus\left[T_{A}, T_{A}\right]$ und definieren eine lineare Abbildung $\sigma$ von $\mathscr{L}$ durch

$$
\sigma\left|T_{A}=-I d ; \quad \sigma\right|\left[T_{A}, T_{A}\right]=I d .
$$

Aus den Multiplikationsregeln in $\mathscr{L}$ (siehe 4.) folgt unmittelbar, dass $\sigma$ ein involutorischer Automorphismus von $\mathscr{L}$ ist. Ferner stehen $T_{A}$ und $\left[T_{A}, T_{A}\right]$ bezüglich der Killingform $\beta$ von $\mathscr{L}$ aufeinander 
senkrecht; denn für $x$ aus $T_{A}$ und $y$ aus $\left[T_{A}, T_{A}\right]$ ist $\beta(x, y)=\beta(\sigma x, \sigma y)=$ $\beta(-x, y)=-\beta(x, y)$. Das Radikal von $\mathscr{L}$ ist $R_{T^{\prime}} \oplus\left[R_{T}, T_{A}\right]$, wobei $R_{T^{\prime}}$ das Radikal von $T_{A}$ bezeichnet ([3], Th. 2, 16). Andrerseits ist das Radikal von $\mathscr{L}$ das orthogonale Komplement von $[\mathscr{L}, \mathscr{L}]$ bezüglich $\beta$ ([1]). Es gilt $[\mathscr{L}, \mathscr{L}]=D^{1} T_{A} \oplus\left[T_{A}, T_{A}\right]$ und da $T_{A}$ und $\left[T_{A}, T_{A}\right]$ bezüglich $\beta$ aufeinander senkrecht stehen, folgt aus Lemma 6 und Satz 2: $\left(D^{1} T_{A}\right)^{\perp}=R_{T^{*}}=R$. Wir zeigen als nächstes: $\left(A^{(2)}\right)^{\perp}=$ $\left(D^{1} T_{A}\right)^{\perp}$. Sei $x$ aus $\left(D^{1} T_{A}\right)^{\perp}$. Es gilt $(x,[u, v, w])=(x, R(u, v) w)=0$ für $u, v, w$ aus $T_{A}$ und daraus folgt $(x, L(u v) w)=-(x, D(u, v) w)$. Da $D(u, v)$ eine Derivation von $A$ ist, folgt

$$
(x, u v \cdot a b)=(x,(u v \cdot a) b+a(u v \cdot b))
$$

und daher

$$
\begin{aligned}
0 & =(x,[a, b, u v])=(x, 2 a b \cdot u v-(b \cdot u v) a-(u v \cdot a) b) \\
& =(x, 3 a b \cdot u v)+(x, u v \cdot a b-a(u v \cdot b)-(u v \cdot a) b) \\
& =3(x, a b \cdot u v) .
\end{aligned}
$$

Da $a, b, u, v$ beliebige Elemente von $A$ waren, ist $x$ aus $\left(A^{(2)}\right)^{\perp}$. Sei andrerseits $x$ aus $\left(A^{(2)}\right)^{\perp}$. Es gilt

$$
(x, a b \cdot u v)=(x \cdot a b, u v)=0 ;
$$

denn die Killingform ist eine assoziative Bilinearform ([4], Th. 7.16). Es folgt $x \cdot a b \in\left(A^{(1)}\right)^{\perp}$ und wegen $A^{(1)} \supset D^{1} T_{A}$ ist $\left(A^{(1)}\right)^{\perp} \subset\left(D^{1} T_{A}\right)^{\perp}=R$, also $x \cdot a b \in R$ für alle $a, b$ aus $A$. Bezeichnen wir mit einem Querstrich das Bild eines Elements aus $A$ unter der kanonischen Abbildung von $A$ nach $A / R$, so gilt $\overline{x \cdot a b}=\bar{x} \cdot \bar{a} \bar{b}=0$ für alle $a, b$ aus $A \cdot \operatorname{Da} A / R=\bar{A}$ halbeinfach und daher direkte Summe einfacher Ideale ist (Kor. $1 \mathrm{zu}$ Satz 2), gilt $\bar{A}^{2}=\bar{A}$ und es folgt $\bar{x} \cdot \bar{A}=0$. Die skalaren Vielfachen von $\bar{x}$ bilden daher ein auflösbares Ideal in $\bar{A}$ und aus der Halbeinfachheit von $\bar{A}$ folgt $\bar{x}=0$ oder $x \in R$.

Sei schon bewiesen, dass $\left(A^{(k)}\right)^{\perp}=R$ für $k \geqq 2$. Wegen $A^{(k+1)} \subset$ $A^{(k)}$ ist $\left(A^{(k+1)}\right)^{\perp} \supset\left(A^{(k)}\right)^{\perp}$. Sei andrerseits $x$ aus $\left(A^{(k+1)}\right)^{\perp}$. Dann ist $(x, a b)=(x a, b)=0$ für $a, b$ aus $A^{(k)}$ und daher $x a$ aus $\left(A^{(k)}\right)^{\perp}=R$. Für das Bild $\bar{x}$ von $x$ unter der kanonischen Abbildung von $A$ auf $\bar{A}=A / R$ gilt dann $\bar{x} \cdot \bar{a}=0$ für $\bar{a}$ aus $\overline{A^{(k)}}=\bar{A}^{(k)}=\bar{A}$; denn $\bar{A}$ ist halbeinfach. Wie oben folgt $\bar{x}=0$ bzw. $x \in R$.

Wir können nun Satz $A$ der Einleitung beweisen. Wenn die Killingform nicht ausgeartet ist, folgt die Halbeinfachheit von $A$ aus [2], Th. 3, p. 71; vgl. auch [4], Th. 7.20. Ist umgekehrt $A$ halbeinfach, so ist wegen $A^{(2)} \subset A$ :

$$
A^{\perp} \subset\left(A^{(2)}\right)^{\perp}=R=0,
$$


also $A^{\perp}=0$, d. h. die Killingform ist nicht ausgeartet.

In [5] bewies Sagle den Satz: Sei $A$ eine einfache Malcev-Algebra endlicher Dimension über einem algebraisch abgeschlossenen Körper der Charakteristik Null, die keine Lie-Algebra ist. Dann ist $A$ enthalte ein Element $x$, sodass $L(x)$ nicht nilpotent ist. $A$ isomorph zu $A^{*}$.

Wie aus Satz $A$ folgt, gibt es in einer einfachen Malcev-Algebra stets ein solches Element; denn sonst wäre $\operatorname{Sp} L(x)^{2}=0$ für alle $x$ aus $A$ und die Killingform wäre identisch Null. Damit ist auch Satz $\mathrm{B}$ bewiesen.

\section{LITERATUR}

1. N. Bourbaki, Algèbres de Lie, Hermann, Paris, 1960.

2. N. Jacobson, Lie Algebras, Interscience, New York-London, 1962.

3. W. G. Lister, A structure theory of Lie triple systems, Trans. Amer. Math. Soc. 72 (1952), 217-242.

4. A. A. Sagle, Malcev algebras, Trans. Amer. Math. Soc. 101 (1961), 426-458.

5. - Simple Malcev algebras over fields of characteristic zero, Pacific J. Math. 12 (1962), 1057-1078.

6. - On derivations of semisimple Malcev algebras, Portugal. Math. 21 (1962), 107-109.

7. R. D. Schafer, An introduction to nonassociative algebras, Lecture notes, Stillwater, 1961.

8. K. Yamaguti, On algebras of totally geodesic subspaces (Lie triple systems), J. Sc. Hiroshima Univ., Ser. A, 21 (1957), 107-113.

Received May 1, 1965. Der Autor dankt Herrn Prof. M. Koecher für viele wertvolle Hinweise bei der Abfassung des Manuskriptes. 


\section{PACIFIC JOURNAL OF MATHEMATICS}

\section{EDITORS}

\author{
H. SAMELSON, \\ Stanford University \\ Stanford, California \\ R. M. BLUMENTHAL \\ University of Washington \\ Seattle, Washington 98105
}

\author{
*J. DugundJI \\ University of Southern California \\ Los Angeles, California 90007 \\ RICHARD ARENS \\ University of California \\ Los Angeles, California 90024
}

\section{ASSOCIATE EDITORS}
E. F. BECKENBACH
B. H. NeUmanN
F. WOLF
K. YOSIDA

\section{SUPPORTING INSTITUTIONS}

\author{
UNIVERSITY OF BRITISH COLUMBIA \\ CALIFORNIA INSTITUTE OF TECHNOLOGY \\ UNIVERSITY OF CALIFORNIA \\ MONTANA STATE UNIVERSITY \\ UNIVERSITY OF NEVADA \\ NEW MEXICO STATE UNIVERSITY \\ OREGON STATE UNIVERSITY \\ UNIVERSITY OF OREGON \\ OSAKA UNIVERSITY \\ UNIVERSITY OF SOUTHERN CALIFORNIA
}

\author{
STANFORD UNIVERSITY \\ UNIVERSITY OF TOKYO \\ UNIVERSITY OF UTAH \\ WASHINGTON STATE UNIVERSITY \\ UNIVERSITY OF WASHINGTON \\ * * * \\ AMERICAN MATHEMATICAL SOCIETY \\ CHEVRON RESEARCH CORPORATION \\ TRW SYSTEMS \\ NAVAL ORDNANCE TEST STATION
}

Mathematical papers intended for publication in the Pacific Journal of Mathematics should be typewritten (double spaced). The first paragraph or two must be capable of being used separately as a synopsis of the entire paper. It should not contain references to the bibliography. Manuscripts may be sent to any one of the four editors. All other communications to the editors should be addressed to the managing editor, Richard Arens at the University of California, Los Angeles, California 90024 .

50 reprints per author of each article are furnished free of charge; additional copies may be obtained at cost in multiples of 50 .

The Pacific Journal of Mathematics is published monthly. Effective with Volume 16 the price per volume (3 numbers) is $\$ 8.00$; single issues, $\$ 3.00$. Special price for current issues to individual faculty members of supporting institutions and to individual members of the American Mathematical Society: $\$ 4.00$ per volume; single issues $\$ 1.50$. Back numbers are available.

Subscriptions, orders for back numbers, and changes of address should be sent to Pacific Journal of Mathematics, 103 Highland Boulevard, Berkeley 8, California.

Printed at Kokusai Bunken Insatsusha (International Academic Printing Co., Ltd.), No. 6, 2-chome, Fujimi-cho, Chiyoda-ku, Tokyo, Japan.

PUBLISHED BY PACIFIC JOURNAL OF MATHEMATICS, A NON-PROFIT CORPORATION

The Supporting Institutions listed above contribute to the cost of publication of this Journal, but they are not owners or publishers and have no responsibility for its content or policies.

* Paul A. White, Acting Editor until J. Dugundji returns. 


\section{Pacific Journal of Mathematics}

\section{Vol. 18, No. 3 \\ May, 1966}

William George Bade and Philip C. Curtis, Jr., Embedding theorems for commutative Banach algebras .......................... 391

Wilfred Eaton Barnes, On the $\Gamma$-rings of Nobusawa ................. 411

J. D. Brooks, Second order dissipative operators ................ 423

Selwyn Ross Caradus, Operators with finite ascent and descent ........ 437

Earl A. Coddington and Anton Zettl, Hermitian and anti-hermitian properties of Green's matrices .......................... 451

Robert Arnold Di Paola, On sets represented by the same formula in distinct consistent axiomatizable Rosser theories ................... 455

Mary Rodriguez Embry, Conditions implying normality in Hilbert space ...........................................

Garth Ian Gaudry, Quasimeasures and operators commuting with convolution ................................... 461

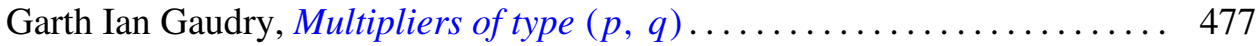

Ernest Lyle Griffin, Jr., Everywhere defined linear transformations affiliated with rings of operators .................................. 489

Philip Hartman, On the bounded slope condition ................ 495

David Wilson Henderson, Relative general position ................ 513

William Branham Jones, Duality and types of completeness in locally

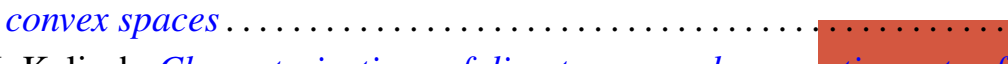

G. K. Kalisch, Characterizations of direct sums and commuting sets of Volterra operators....

Ottmar Loos, Über eine Beziehung zwischen Malcev-Algebren und Lietripelsystemen

Ronson Joseph Warne, A class of bisimple inverse semigroups . . . 